\title{
Utility of the immunohistochemical detection of FLI-1 expression in round cell and vascular neoplasm using a monoclonal antibody
}

\author{
Sabrina Rossi ${ }^{1}$, Enrico Orvieto ${ }^{1}$, Alberto Furlanetto ${ }^{1}$, Licia Laurino ${ }^{1}$, Vito Ninfo $^{2}$ and \\ Angelo P Dei Tos ${ }^{1}$ \\ ${ }^{1}$ Department of Pathology, Regional Hospital, Treviso, Italy and ${ }^{2}$ Department of Pathology, University of \\ Padua School of Medicine, Padua, Italy
}

\begin{abstract}
FLI-1 nuclear transcription factor has been proposed as a useful tool in the differential diagnosis of small round cell sarcomas. Recently, FLI-1 has been reported as the first nuclear marker of endothelial differentiation. However, its clinical use has been hampered by major interpretation problems, due to the presence of background staining as well as staining variation between different lots of the same antiserum. In this study, a novel monoclonal antibody raised against the carboxyl terminal of the FLI-1 protein (clone GI146-222, BD Pharmingen) was tested in a series of small round cell and vascular neoplasms. Furthermore, in order to assess FLI-1 specificity, we analyzed its expression in a series of common epithelial and nonepithelial malignancies. In total, 15 Ewing's sarcomas, 10 rhabdomyosarcomas, 5 desmoplastic small round cell tumors, 10 synovial sarcomas, 10 high-grade pleomorphic sarcomas, 10 malignant melanomas, 5 Merkel's carcinomas, 10 colonic adenocarcinomas, 10 breast carcinomas, 10 lung adenocarcinomas, 20 angiosarcomas, 5 epithelioid hemangioendotheliomas, 10 Kaposi's sarcomas and 10 benign hemangiomas, were stained. A strong FLI-1 immunoreactivity was detected in all Ewing's sarcomas and vascular neoplasms, highlighting the high sensitivity of FLI-1 monoclonal antibody. However, 2/5 Merkel's carcinomas and 1/10 malignant melanomas showed a strong nuclear immunostaining, suggesting that FLI-1 may not be so helpful in the differential diagnosis of cutaneous Ewing's sarcoma. In addition, a weak immunoreactivity was found in $3 / 5$ Merkel cell carcinomas, $3 / 10$ synovial sarcomas, 5/10 malignant melanomas, $6 / 10$ lung adenocarcinomas and in 1/10 breast carcinomas. In contrast, all the rhabdomyosarcomas, desmoplastic small round cell tumors, high-grade pleomorphic sarcomas and colonic adenocarcinomas tested were negative. Importantly, in contrast with previous studies, no background staining was observed. Our results indicate that FLI-1 monoclonal antibody can be reliably applied to the differential diagnosis of small round cell neoplasms of soft tissue, and confirm its important role as nuclear marker of endothelial differentiation, mainly helpful in those cases in which technical artifacts are seen by using the traditional membranous and cytoplasmic endothelial markers.
\end{abstract}

Modern Pathology (2004) 17, 547-552, advance online publication, 5 March 2004; doi:10.1038/modpathol.3800065

Keywords: FLI-1; immunohistochemistry; round cell tumors; vascular tumors

FLI-1 is a member of ETS genes, which encode for a family of transcription factors, defined by a highly conserved DNA-binding domain. ${ }^{1}$ Its clinical role is most apparent in the human Ewing's sarcoma/ primitive neuroectodermal tumor (ES/PNET) category, wherein FLI-1 is the target of a characteristic

Correspondence: Dr AP Dei Tos, MD, Departments of Pathology and Oncology, Regional Hospital, Piazza Ospedale 1, I-31100, Treviso, Italy.

E-mail: apdeitos@ulss.tv.it

Presented in part at the 92nd Meeting of the United States and Canadian Academy of Pathology, Washington, DC, March 22-28, 2003.

Received 01 August 2003; revised 10 December 2003; accepted 16 December 2003; published online 5 March 2004 balanced chromosomal translocation $t(11 ; 22)$ (q24:q12) which results in the production of the EWS/FLI-1 fusion gene. The resultant EWS/FLI-1 chimeric protein includes the $\mathrm{N}$-terminal transactivation domain of EWS and the C-terminal DNAbinding domain of FLI- $1 .^{2}$ The EWS/FLI-1 chimeric protein binds DNA with the same affinity as FLI-1, but EWS/FLI-1 transcriptional activation of the target genes is much more efficient. ${ }^{3}$

ES/PNET belongs to a heterogeneous group of mesenchymal neoplasms featuring round cell morphology and therefore collectively known as small round cell sarcomas. Alveolar rhabdomyosarcoma (RMS), desmoplastic small round cell tumor (DSRCT), mesenchymal chondrosarcoma and poorly differentiated synovial sarcoma (SS) also belong to 
this group. Immunohistochemistry plays a key role in the differential diagnosis of round cell tumors and CD99 is a very sensitive marker of ES/ PNET. However, its expression is not limited to these tumors. CD99 immunoreactivity has been observed in RMS, ${ }^{4} \mathrm{SS},{ }^{5-7}$ lymphoblastic lymphoma, ${ }^{8}$ mesenchymal chondrosarcoma, ${ }^{9,10}$ Merkel cell carcinoma (MCC) ${ }^{11}$ and has been also reported in rare cases of Wilms' tumor, ${ }^{12}$ small cell osteosarcoma $^{13}$ and DSRCT. ${ }^{14,15}$ Thus, FLI-1 has been recently proposed as an additional immunohistochemical marker of ES/PNET, along side the traditional CD99. ${ }^{16-18}$

Moreover, as FLI-1 protein expression is consistently found in endothelial cells as well as neoplasm thereof, ${ }^{18}$ FLI-1 has been also proposed as a nuclear marker of endothelial differentiation. ${ }^{19}$

In the previous series, the use of a polyclonal antiserum has led to major interpretation problems, due to the presence of background staining as well as staining variation between different lots of the same antiserum. ${ }^{18,19}$ As a consequence, FLI-1 immunohistochemistry has never become popular. In the present study, a novel monoclonal antibody raised against the carboxyl terminal of the FLI-1 protein (clone GI146-222, BD Pharmingen) was tested. FLI-1 expression was investigated in a series of both small round cell neoplasms and vascular neoplasms. In addition, in order to assess FLI-1 specificity, its expression was analyzed in a series of common epithelial and non-epithelial malignancies.

\section{Materials and methods}

Formalin-fixed, paraffin-embedded archival tissue from 15 ES/PNET, 10 RMS, five DSRCT, 10 SS, 10 high-grade pleomorphic sarcomas (PS), 10 malignant melanomas (MM), five MCC, 10 colonic adenocarcinomas (CA), 10 breast carcinomas (BC), 10 lung adenocarcinomas (LA), 20 angiosarcomas (AS), five epithelioid hemangioendotheliomas (EHE), 10 Kaposi's sarcomas (KS) and 10 benign hemangiomas (BH), were immunostained with a monoclonal antibody raised against the carboxyl terminal of the FLI-1 protein (clone G146-222, BD Pharmingen, dilution 1:50). Immunostains were performed using a sensitive polymer based revelation system (Envision Plus, DakoCytomation, Glostrup Denmark). Heat-induced antigen retrieval was performed by incubating the slides for $40^{\prime}$ at $98^{\circ} \mathrm{C}$ in a waterbath using an $8.5 \mathrm{pH}$ EDTA buffer. All immunostains were performed on an automated immunostainer (Dako Autostainer, DakoCytomation). Positivity was scored using a four-tiered scale $(1+=<25 \%$ positive cells; $2+=26-50 \% ; \quad 3+=51-75 \% ; \quad 4+=76-100 \%)$. Normal endothelium served as built-in positive control in all cases. Only nuclear immunoreactivity was evaluated.

\section{Results}

All ES/PNET (Figures 1a and b) as well as all vascular tumors (Figures 1c and d), exhibited a $4+$,

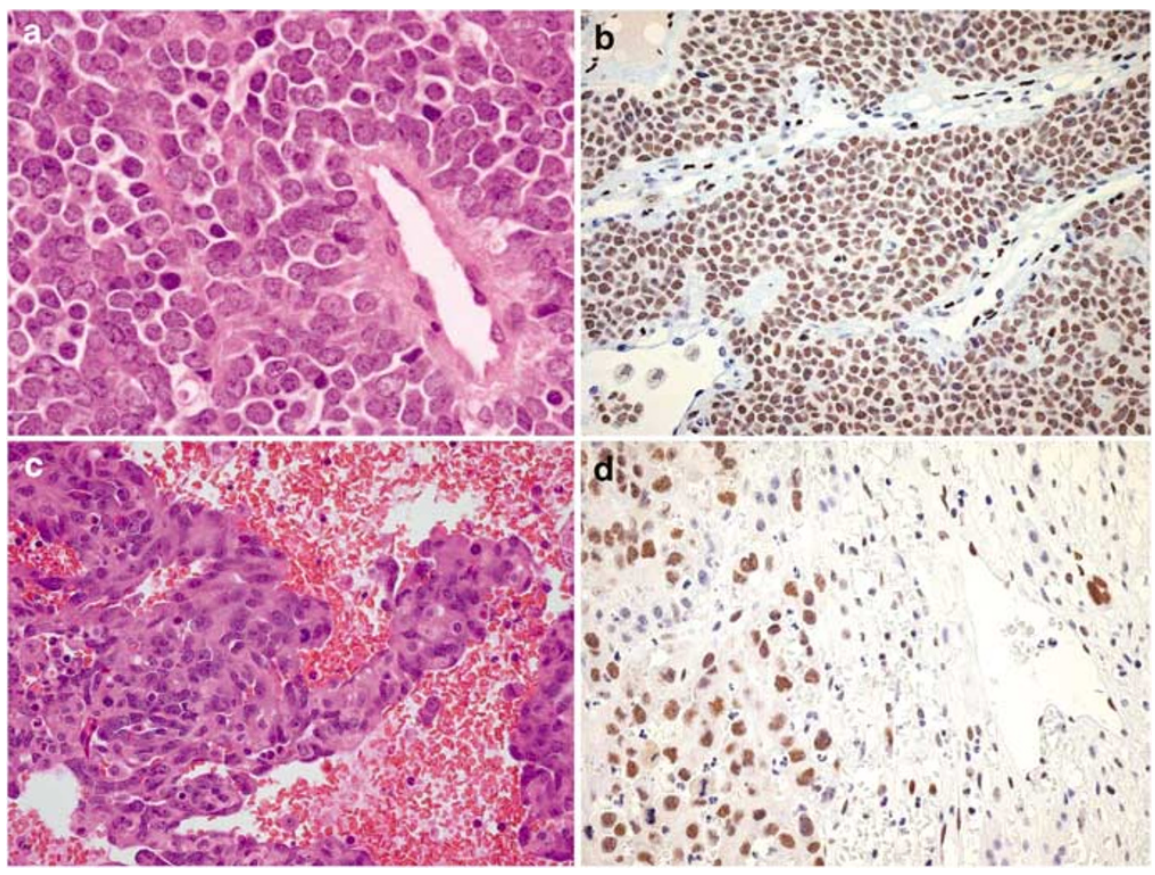

Figure 1 (a) Ewing's sarcoma/PNET represents the prototype of small round cell mesenchymal malignancies ( $\times$ 400); (b) FLI-1 monoclonal antibody decorates all Ewing's sarcoma cell nuclei $(\times 200)$; $(\mathbf{c})$ epithelioid angiosarcoma may exhibit a solid growth pattern that mimics carcinoma ( $\times 400)$; (d) all cases of epithelioid angiosarcoma exhibited strong FLI-1 immunoreactivity $(\times 400)$; Importantly, FLI-1 immunopositivity is detectable in normal endothelium as well as in benign and malignant vascular neoplasm. 


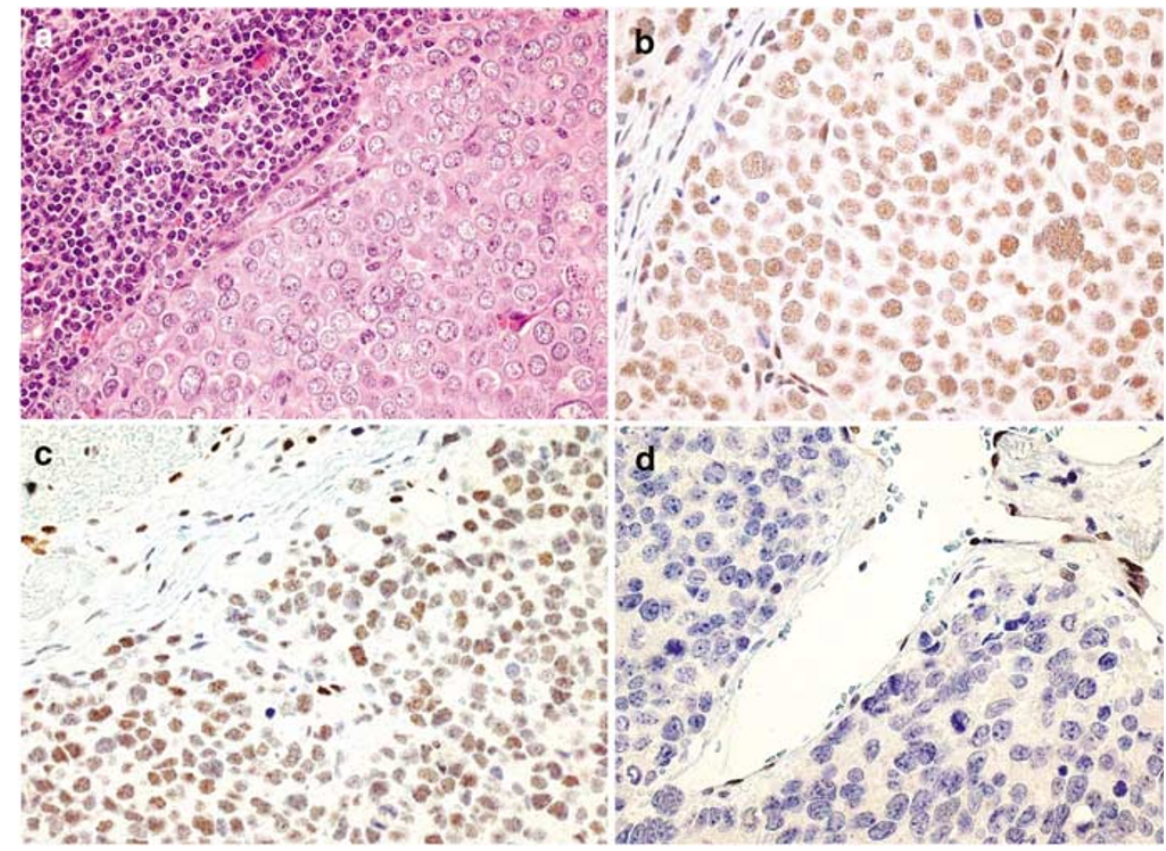

Figure 2 (a) Merkel cell carcinoma may represent an important diagnostic challenge when dealing with round cell neoplasm arising in adults ( $\times 200)$; (b) FLI-1 immunopositivity was observed in all Merkel cell carcinomas tested $(\times 400)$; (c) exceptionally, malignant melanoma exhibited strong FLI-1 immunoreactivity; (d) infiltrating ductal breast carcinoma showing no FLI-1 staining. Endothelial cells serve as built-in positive control $(\times 400)$.

Table 1 Immunohistochemical results

\begin{tabular}{lcc}
\hline Tumor & FLI-1 Immunopositivity & Score \\
\hline ES & $15 / 15(100 \%)$ & $4+$ \\
RMS & $0 / 10(0 \%)$ & - \\
DSRCT & $0 / 5(0 \%)$ & - \\
SS & $3 / 10(30 \%)$ & $1-2+$ \\
PS & $0 / 10(0 \%)$ & $2+$ \\
MM & $6 / 10(60 \%)$ & $3+$ \\
MCC & $5 / 5(100 \%)$ & $1-2+$ \\
LA & $6 / 10(60 \%)$ & $2+$ \\
BC & $1 / 10(10 \%)$ & - \\
CA & $0 / 10(0 \%)$ & $4+$ \\
EHE & $5 / 5(100 \%)$ & $4+$ \\
KS & $10 / 10(100 \%)$ & $4+$ \\
BH & $10 / 10(100 \%)$ & $4+$ \\
AS & $20 / 20(100 \%)$ & \\
& &
\end{tabular}

ES, Ewing's sarcoma; RMS, rhabdomyosarcoma; DSRCT, desmoplastic small round cell tumor; SS, synovial sarcoma; PS, highgrade pleomorphic sarcoma; MM, malignant melanoma; MCC, Merkel' cell carcinoma; CA, colonic adenocarcinoma; BC, breast carcinoma; LA, lung adenocarcinoma; AS, angiosarcoma; EHE, epithelioid hemangioendothelioma; KS, Kaposi’s sarcoma; BH, benign hemangiomas.

strong nuclear immunoreactivity. Strong immunoreactivity was also found in 2/5 MCC (Figures 2a and b) and in 1/10 MM (Figure 2c). Weak nuclear immunoreactivity, ranging between $1+$ and $2+$ was observed in 3/5 MCC, 5/10 MM, 3/10 SS, 6/10 LA, 1/10 BC (Figure 2d). All RMS, DSRCT, PS and CA tested were negative, as shown in Table 1. No cytoplasmic background staining was observed.

\section{Discussion}

FLI-1 gene was first identified in Friend virusinduced erythroleukemia at the site of retroviral integration. ${ }^{20}$ Subsequent analyses in different models of virus-induced lymphoma/leukemia have shown FLI-1 gene rearrangements at the site of proviral integration. ${ }^{1}$ FLI-1 gene belongs to the human Ets family of transcription factors and localizes at the Ets locus on human chromosome 11q23. ${ }^{1}$ Interestingly, FLI-1 expression has been shown to be critical for hematopoiesis and early vessels development, as demonstrated in knockout mice $^{21}$ and zebrafish. ${ }^{22}$ Moreover, FLI-1 protein expression has been found in endothelial ${ }^{18}$ and hemopoietic cells, as T-lymphocytes and megacaryocytes. ${ }^{1}$

In most of the human ES/PNET, FLI-1 is the target of a characteristic chromosomal balanced translocation $\mathrm{t}(11 ; 22)$ (q24:q12), which results in the production of EWS/FLI-1 fusion gene. In the remaining cases of ES/PNET, the EWS gene is rearranged with other partners of the Ets oncogene family. Interestingly, ES/PNET cases harboring the EWS/FLI-1 transcript seem to have a better prognosis than those containing other EWS/ETS transcripts. ${ }^{23}$ Furthermore, since the copy number of $1 \mathrm{q}$ and $16 \mathrm{q}$ may identify a group of ES/PNET patients with a worse prognosis, the importance to assess the presence of additional cytogenetic aberrations has been recently highlighted. ${ }^{24}$

A few mechanisms underlying EWS/FLI-1 oncogenetic activity have been hypothesized. EWS/FLI-1 
but not FLI-1 alone induces malignant transformation in NIH3T3 cells. ${ }^{25-27}$ Interestingly, this phenotype may be reversed by using a transcriptional repressor, which inhibits only the transcriptional activity of EWS/FLI-1, confirming that its oncogenic activity is related to the aberrant expression of specific EWS/FLI-1 target genes. ${ }^{28}$ However, since its transforming activity is not only dependent upon the DNA-binding domain, it has been suggested that a protein-protein interaction may represent an additional FLI-1 oncogenetic mechanism. ${ }^{29}$ Interestingly, up-regulation of $\mathrm{c}-m y c$ is observed in the cell lines expressing EWS/FLI- $1{ }^{30}$ In contrast, the TGF- $\beta$ pathway and p57 protein, which are involved in the cell-cycle inhibition, have been shown to be downregulated. ${ }^{30}$

A polyclonal antiserum raised against the Cterminus of the FLI-1 protein has been previously reported as a useful tool in distinguishing ES/PNET from other round cell tumors. ${ }^{18}$ However, its clinical use has been hampered by major interpretation problems, due to the presence of background staining as well as staining variation between different lots of the same antiserum. In the current study, FLI-1 expression was investigated in 15 cases of ES/PNET and a strong nuclear immunoreactivity was detected in all cases. Since in the previous series FLI-1 was expressed in $71 \%^{18}$ and $92 \%^{17}$ of ES/PNET tested, the higher sensitivity of this FLI-1 monoclonal antibody is apparent. It may be correctly argued that some of the cases in Folpe's study ${ }^{18}$ might be characterized by other EWS/ETS translocations. However, it should be noted that in LlombartBosch's series, ${ }^{17}$ RT-PCR analysis demonstrated the presence of EWS/FLI-1 translocation in all the cases included.

No FLI-1 expression was found in RMS and DSRCT, suggesting that FLI-1 may be used in the differential diagnosis of round small cell tumors. Importantly, lower levels of FLI-1 immunopositivity were observed in a significant proportion of SS, MM as well as common epithelial malignancies. Even if, with the exceptions of one case of $\mathrm{MM}$ and two cases of MCC, the intensity of the nuclear staining ranged from weak to moderate, this underlines the importance of evaluating immunohistochemical results in context with morphology and in panel with other immunoreagents.

The detection of FLI-1 expression in MCC, limits its role in the differential diagnosis of cutaneous $E^{31-33}$ and expands further the morphologic overlap between MCC and those rare examples of ES arising primarily in the skin.

It has also to be emphasized that the immunohistochemical detection of proteins involved in the formation of fusion transcripts may represent a faster as well as cheaper mean to predict the presence of the underlying chromosome abnormalities. This has been proved true for alk-1 immunostaining in both anaplastic large cell lymphomas ${ }^{34-36}$ and inflammatory myofibroblastic tumors. ${ }^{37,38}$
Immunohistochemical detection of WT1 in desmoplastic small round cell tumor ${ }^{39,40}$ and of TFE3 in alveolar soft part sarcoma ${ }^{41,42}$ represent additional examples of this approach.

FLI-1 clinical use is not limited to the differential diagnosis of small round cell tumors. Since FLI-1 protein is present in normal endothelial cells, ${ }^{18}$ its expression has been recently investigated in a series of endothelial neoplasms. ${ }^{19}$ In Folpe's study, the sensitivity of the FLI-1 polyclonal antibody was about $90 \%$ in AS and EHE and $100 \%$ in BH and KS. In our series, strong immunoreactivity was detected in all the endothelial neoplasms, including AS and EHE, suggesting that, even in this setting, FLI-1 monoclonal antibody appears to exhibit a higher sensitivity than the polyclonal antiserum. Our results confirm the important role of FLI-1 immunohistochemistry as a very reliable nuclear marker of endothelial differentiation, mainly helpful in those cases in which the evaluation of the traditional membranous and cytoplasmic endothelial markers such as CD31, CD34 and FVIII-RA is hampered by technical artifacts. Importantly, FLI-1 is totally useless in distinguishing benign from malignant vascular proliferations. In fact, strong immunoreactivity is present in normal endothelial as well as in any neoplasm thereof.

In contrast with a previous series, ${ }^{18}$ we found a weak/moderate FLI-1 expression in $60 \%$ of LA and $10 \%$ of BC. Interestingly, our results are consistent with the previous observation that FLI-1 is expressed in normal epithelia, as breast epithelium and cutaneous eccrine glands. ${ }^{19}$

In conclusion, our results indicate that FLI-1 monoclonal antibody, when evaluated in context with morphology, can be reliably applied to the differential diagnosis of small round cell neoplasms of soft tissue. In addition, FLI-1 currently emerges as the most sensitive marker of endothelial differentiation.

\section{Acknowledgement}

We thank Ms Serena Chinellato for her excellent technical support.

\section{References}

1 Truong AH, Ben-David Y. The role of FLI-1 in normal cell function and malignant transformation. Oncogene 2000;19:6482-6489.

2 Delattre O, Zucman J, Plougastel B, et al. Gene fusion with an ETS DNA-binding domain caused by chromosome translocation in human tumours. Nature 1992;359:162-165.

3 May WA, Denny CT. Biology of EWS/FLI and related fusion genes in Ewing's sarcoma and primitive neuroectodermal tumor. Curr Top Microbiol Immunol 1997;220:143-150. 
4 Stevenson AJ, Weber MP, Trudel R, et al. Monitoring furosemide in racehorses participating in an EIPH program. J Vet Pharmacol Ther 1994;17:163-168.

5 Dei Tos AP, Wadden C, Calonje E, et al. Immunohistochemical demonstration of glycoprotein p30/ 32(MIC2) (CD99) in synovial sarcoma: a potential cause of diagnostic confusion. Appl Immunohistochem 1995;3:168-173.

6 Folpe AL, Schmidt RA, Chapman D, et al. Poorly differentiated synovial sarcoma: immunohistochemical distinction from primitive neuroectodermal tumors and high-grade malignant peripheral nerve sheath tumors. Am J Surg Pathol 1998;22:673-682.

7 van de Rijn M, Barr FG, Xiong QB, et al. Poorly differentiated synovial sarcoma: an analysis of clinical, pathologic, and molecular genetic features. Am J Surg Pathol 1999;23:106-112.

8 Riopel M, Dickman PS, Link MP, et al. MIC2 analysis in pediatric lymphomas and leukemias. Hum Pathol 1994;25:396-399.

9 Granter SR, Renshaw AA, Fletcher CD, et al. CD99 reactivity in mesenchymal chondrosarcoma. Hum Pathol 1996;27:1273-1276.

10 Devaney KO, Ferlito A, Silver CE. Cartilaginous tumors of the larynx. Ann Otol Rhinol Laryngol 1995;104:251-255.

11 Nicholson SA, McDermott MB, Swanson PE, et al. CD99 and cytokeratin-20 in small-cell and basaloid tumors of the skin. Appl Immunohistochem Mol Morphol 2000;8:37-41.

12 Folpe AL, Patterson K, Gown AM. Antibodies to desmin identify the blastemmal component of nephroblastoma. Mod Pathol 1997;10:895-900.

13 Devaney K, Vinh TN, Sweet DE. Small cell osteosarcoma of bone: an immunohistochemical study with differential diagnostic considerations. Hum Pathol 1993;24:1211-1225.

14 Ordi J, de Alava E, Torne A, et al. Intraabdominal desmoplastic small round cell tumor with EWS/ERG fusion transcript. Am J Surg Pathol 1998;22: 1026-1032.

15 Ordonez NG. Desmoplastic small round cell tumor: II: an ultrastructural and immunohistochemical study with emphasis on new immunohistochemical markers. Am J Surg Pathol 1998;22:1314-1327.

16 Nilsson G, Wang M, Wejde J, et al. Detection of EWS/ FLI-1 by immunostaining An adjunctive tool in diagnosis of Ewing's sarcoma and primitive neuroectodermal tumor on cytological samples and paraffinembedded archival material. Sarcoma 1999;3:25-32.

17 Llombart-Bosch A, Navarro S. Immunohistochemical detection of EWS and FLI-1 proteinss in Ewing sarcoma and primitive neuroectodermal tumors: comparative analysis with CD99 (MIC-2) expression. Appl Immunohistochem Mol Morphol 2001;9:255-260.

18 Folpe AL, Hill CE, Parham DM, et al. Immunohistochemical detection of FLI-1 protein expression: a study of 132 round cell tumors with emphasis on CD99positive mimics of Ewing's sarcoma/primitive neuroectodermal tumor. Am J Surg Pathol 2000;24: 1657-1662.

19 Folpe AL, Chand EM, Goldblum JR, et al. Expression of FLI-1, a nuclear transcription factor, distinguishes vascular neoplasms from potential mimics. Am J Surg Pathol 2001;25:1061-1066.

20 Ben-David Y, Giddens EB, Letwin K, et al. Erythroleukemia induction by Friend murine leukemia virus: insertional activation of a new member of the ets gene family, FLI-1, closely linked to c-ets-1. Genes Dev 1991;5:908-918.

21 Hart A, Melet F, Grossfeld P, et al. FLI-1 is required for murine vascular and megakaryocytic development and is hemizygously deleted in patients with thrombocytopenia. Immunity 2000;13:167-177.

22 Brown LA, Rodaway AR, Schilling TF, et al. Insights into early vasculogenesis revealed by expression of the ETS-domain transcription factor FLI-1 in wild-type and mutant zebrafish embryos. Mech Dev 2000;90:237-252.

23 de Alava E, Kawai A, Healey JH, et al. EWS-FLI1 fusion transcript structure is an independent determinant of prognosis in Ewing's sarcoma. J Clin Oncol 1998;16:1248-1255.

24 Hattinger CM, Potschger U, Tarkkanen M, et al. Prognostic impact of chromosomal aberrations in Ewing tumours. Br J Cancer 2002;86:1763-1769.

25 Thompson AD, Teitell MA, Arvand A, et al. Divergent Ewing's sarcoma EWS/ETS fusions confer a common tumorigenic phenotype on NIH3T3 cells. Oncogene 1999;18:5506-5513.

26 May WA, Arvand A, Thompson AD, et al. EWS/FLI1induced manic fringe renders NIH 3T3 cells tumorigenic. Nat Genet 1997;17:495-497.

27 Arvand A, Welford SM, Teitell MA, et al. The COOHterminal domain of FLI-1 is necessary for full tumorigenesis and transcriptional modulation by EWS/FLI1. Cancer Res 2001;61:5311-5317.

28 Chan D, Wilson TJ, Xu D, et al. Transformation induced by Ewing's sarcoma associated EWS/FLI-1 is suppressed by KRAB/FLI-1. Br J Cancer 2003;88: 137-145.

29 Jaishankar S, Zhang J, Roussel MF, et al. Transforming activity of EWS/FLI is not strictly dependent upon DNA-binding activity. Oncogene 1999;18:5592-5597.

30 Dauphinot L, De Oliveira C, Melot T, et al. Analysis of the expression of cell cycle regulators in Ewing cell lines: EWS-FLI-1 modulates p57KIP2and c-Myc expression. Oncogene 2001;20:3258-3265.

31 Chow E, Merchant TE, Pappo A, et al. Cutaneous and subcutaneous Ewing's sarcoma: an indolent disease. Int J Radiat Oncol Biol Phys 2000;46:433-438.

32 Hasegawa SL, Davison JM, Rutten A, et al. Primary cutaneous Ewing's sarcoma: immunophenotypic and molecular cytogenetic evaluation of five cases. Am J Surg Pathol 1998;22:310-318.

33 Banerjee SS, Agbamu DA, Eyden BP, et al. Clinicopathological characteristics of peripheral primitive neuroectodermal tumour of skin and subcutaneous tissue. Histopathology 1997;31:355-366.

34 Pittaluga S, Wiodarska I, Pulford $\mathrm{K}$, et al. The monoclonal antibody ALK1 identifies a distinct morphological subtype of anaplastic large cell lymphoma associated with 2p23/ALK rearrangements. Am J Pathol 1997;151:343-351.

35 Cataldo KA, Jalal SM, Law ME, et al. Detection of $t(2 ; 5)$ in anaplastic large cell lymphoma: comparison of immunohistochemical studies, FISH, and RT-PCR in paraffin-embedded tissue. Am J Surg Pathol 1999;23:1386-1392.

36 Pulford K, Lamant L, Morris SW, et al. Detection of anaplastic lymphoma kinase (ALK) and nucleolar protein nucleophosmin (NPM)-ALK proteins in normal and neoplastic cells with the monoclonal antibody ALK1. Blood 1997;89:1394-1404. 
37 Cessna MH, Zhou H, Sanger WG, et al. Expression of ALK1 and p80 in inflammatory myofibroblastic tumor and its mesenchymal mimics: a study of 135 cases. Mod Pathol 2002;15:931-938.

38 Cook JR, Dehner LP, Collins $\mathrm{MH}$, et al. Anaplastic lymphoma kinase (ALK) expression in the inflammatory myofibroblastic tumor: a comparative immunohistochemical study. Am J Surg Pathol 2001;25:13641371.

39 Hill DA, Pfeifer JD, Marley EF, et al. WT1 staining reliably differentiates desmoplastic small round cell tumor from Ewing sarcoma/primitive neuroectodermal tumor. An immunohistochemical and molecular diagnostic study. Am J Clin Pathol 2000;114:345-353.
40 Lae ME, Roche PC, Jin L, et al. Desmoplastic small round cell tumor: a clinicopathologic, immunohistochemical, and molecular study of 32 tumors. Am J Surg Pathol 2002;26:823-835.

41 Ladanyi M, Lui MY, Antonescu CR, et al. The $\operatorname{der}(17) t(X ; 17)(p 11 ; q 25)$ of human alveolar soft part sarcoma fuses the TFE3 transcription factor gene to ASPL, a novel gene at 17q25. Oncogene 2001;20: 48-57.

42 Argani P, Lal P, Hutchinson B, et al. Aberrant nuclear immunoreactivity for TFE3 in neoplasms with TFE3 gene fusions: a sensitive and specific immunohistochemical assay. Am J Surg Pathol 2003;27: 750-761. 